\title{
FACTORES DE DESERCIÓN ESTUDIANTIL EN LA UNIVERSIDAD SURCOLOMBIANA
}

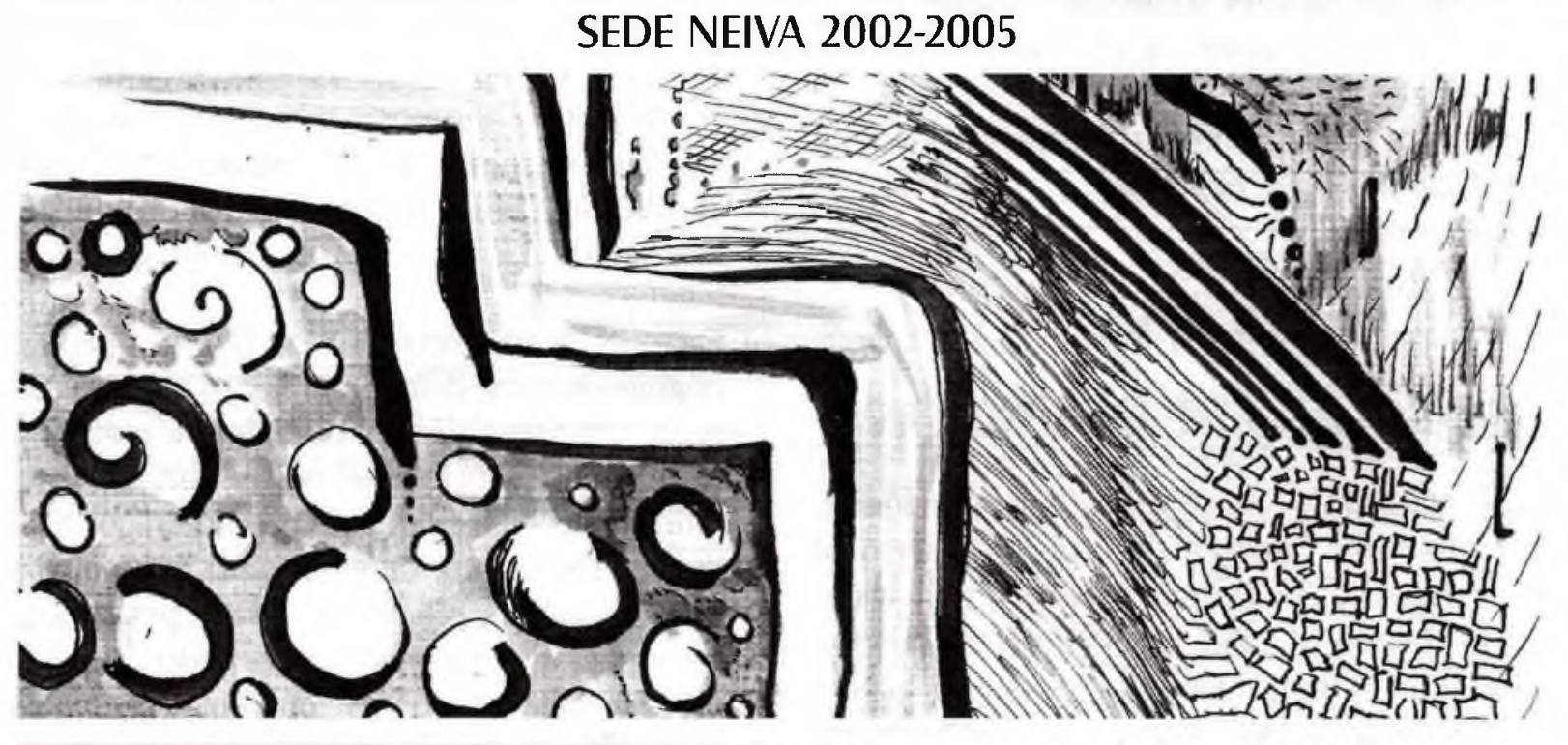

GUILLERMO SÁNCHEZ AMAYA MAGISTER EN PLANEACIÓN DEL DESARROLLO URBANO REGIONAL

WISBERTO NAVARRO SALCEDO MAGISTER EN FILOSOFÍA wisberto@usco.edu.co ALEJANDRO DAVID GARCIA VALENCIA MAGISTER DESARROLLO HUMANO Y PEDAGOGIAS ACTIVAS adavidgarciav@hotmail.com PROFESORES DEPARTAMENTO DE PSICOPEDAGOGÍA FACULTAD DE EDUCACIÓN UNIVERSIDAD SURCOLOMBIANA - USCO

\section{RESUMEN}

En la universidad Surcolombiana en sus 35 años de existencia, el retiro de estudiantes no se ha auscultado ni teórica ni prácticamente y es verdaderamente preocupante que de 100 jóvenes que logran acceder a ésta, cerca de 40 la abandonen, sin culminar su respectivo ciclo académico. Ante tal situación esta investigación se planteó como objetivo central la identificación, análisis, determinación y clasificación de los factores de deserción que presenta la USCO en el periodo 2002-2005.

Metodológicamente es una investigación expostfacto, con un análisis cuantitativo y cualitativo, que aborda el fenómeno de la Deserción Universitaria en la Universidad Surcolombiana, desde una doble perspectiva: la primera de carácter externo; referida al orden socioeconómico; y la segunda de carácter interno; que considera factores académicos.

\section{LA DESERCIÓN ESTUDIANTIL: UN PROBLEMA SOCIAL}

D e acuerdo al ICFES, las cifras de deserción universitaria, entre 1990 y 1999, oscilaban entre $68.14 \%$ y $47.68 \%$; así mismo, el estudio "Situación de la educación básica, media y superior en Colombia" (2003) realizado por la Fundación Restrepo Barco muestra que en nuestro país el mayor número de estudiantes que desertan, lo hacen por razones 
ajenas a ellos y a la Institución; el abandono obedece mas bien a factores socio-económicos. Además, Cecilia Rincón (2004), entiende la deserción como un fenómeno multicausal, en el que confluyen factores subjetivos, factores socioeconómicos como pobreza, inequidad, violencia y marginalidad social; como también, factores propios de la relaciones docente-estudiante y estudiante-institución educativa.

\section{HACIA UN NUEVO CONCEPTO DE DESERCIÓN}

Páramo, Gabriel; Correa, Carlos (1999) consideran que la deserción obedece a una decisión personal del estudiante y por tanto no obedece a un retiro forzoso debido al bajo rendimiento académico o por asuntos disciplinares. Esto significa que la deserción es un asunto voluntario que va creciendo y reforzándose en el interior del sujeto, quien lo manifiesta en la decisión definitiva, para bien o para mal de él mismo y de su entorno.

Jewsbury, Haefeli (2000) citando a Elbaum complementa lo anterior cuando afirma que: "la deserción presupone una conflictividad externa procesada a lo largo de un tránsito de autojustificación. El que abandona primero suele sentirse abandonado por la institución. Se inicia con una ruptura previa espacio-temporal dentro del aula y la relación con el resto de los compañeros se hace mas distante y ajena".

Teniendo en cuenta las apreciaciones anteriores se puede entender por abandono académico al retiro forzado o voluntario que el estudiante hace de un programa académico o de la universidad. Esto lleva a definir Deserción como el abandono académico pero voluntario que el estudiante hace de un programa o de la universidad y mortalidad académica como el retiro forzoso.

\section{CAUSAS DE LA DESERCIÓN ESTUDIANTIL UNIVERSITARIA}

Álvarez, J. M. (1997:49), citando a José Baquero, asocia a cuatro factores las causas principales de la deserción estudiantil universitaria, que son:

a) Factores personales: constituidos por motivos psicológicos, que comprenden aspectos motivacionales, emocionales, desadaptación e insatisfacción de expectativas; motivos sociológicos, debidos a influencias familiares $y$ de otros grupos como los amigos, condiscípulos, vecinos; y otros motivos no clasificados como la edad, salud, fallecimiento, entre otros.

b) Factores académicos: dados por problemas cognitivos como bajo rendimiento académico, repitencia, ausencia de disciplina y métodos de estudio; deficiencias universitarias como dificultades en los programas académicos que tienen que ver con la enseñanza tradicional, insatisfacción académica generada por la falta de espacios pedagógicos adecuados para el estudio, falta de orientación profesional que se manifiesta en una elección inadecuada de carrera o institución y ausencia de aptitud académica.

c) Factores socio-económicos: generados por bajos ingresos familiares, desempleo, falta de apoyo familiar, incompatibilidad de horario entre trabajo y estudio.

d) Factores Institucionales: causados por el cambio de institución, deficiencia administrativa, influencia negativa de los docentes y otras personas de la institución, programas académicos obsoletos y rígidos, baja calidad educativa.

\section{LA DESERCIÓN EN LA USCO}

De acuerdo al cuadro 01 (Deserción y Retención 2002-2005), se puede observar que los programas de: Ingeniería Agrícola, Educación Artística y Ciencias Naturales son los que presentan el índice más alto de deserción; en el caso de Ingeniería Agrícola y ciencias Naturales obedecen a que estos programas se toman como "trampolín" para ingresar a Ingeniería de Petróleos o Electrónica y Medicina por su fuerte componente en ciencias básicas (matemáticas, física, química y biología), a diferencia de Educación Artística donde el mayor problema radica en 
deficiencias en el uso de estrategias cognitivas lo que hace que estos estudiantes presenten dificultades de motivación y compromiso con el programa. Muchos de los estudiantes que ingresan se proyectan como artistas y no como licenciados, lo cual lleva a subvalorar el componente pedagógico. Además la unificación del programa (Licenciatura en artes visuales, música y arte escénico) generó inconformidad ya que los estudiantes se vieron obligados a estudiar componentes artísticos ajenos a sus intereses.

Una característica en común que presenta este tipo de población desertora en los programas anteriormente aludidos es que no cuentan con una situación económica estable, hecho que conduce a la desmotivación y falta de compromiso con sus responsabilidades académicas que impide la realización de su proyecto de vida como profesional.

El caso contrario se aprecia en los programas de Medicina, Enfermería e Ingeniería de Petróleos, programas que presentan el índice más bajo de deserción de los 17. En el caso de Medicina e Ingeniería de Petróleos son los programas que exigen el mayor porcentaje de ICFES para ingresar a la Universidad, lo cual garantiza que sean estudiantes con unas competencias cognitivas idóneas y con una claridad en su vocación profesional; algo similar sucede con Enfermería, pues generalmente las personas que se presentan al área de salud tienen bien definido lo que desean estudiar y lo culminan generalmente a pesar de sus limitaciones económicas.

\begin{tabular}{|c|c|c|c|c|}
\hline PROGRAMA & 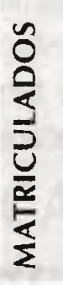 & 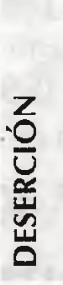 & $\frac{Z}{0}$ & 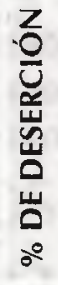 \\
\hline ACUICULTURA & 71 & 36 & 35 & 51 \\
\hline INGE. AGRÍCOLA & 40 & 26 & 14 & 65 \\
\hline EDU. ARTÍSTICA & 42 & 25 & 17 & 60 \\
\hline CONTADURÍA & 50 & 24 & 26 & 48 \\
\hline CIENCIAS NATURALES & 40 & 22 & 18 & 55 \\
\hline ADMON EMPRESAS & 50 & 21 & 29 & 42 \\
\hline LENGUA CASTELLANA & 42 & 21 & 21 & 50 \\
\hline
\end{tabular}

$\begin{array}{lllll}\text { EDU. FÍSICA } & 43 & 18 & 25 & 42 \\ \text { INGE. ELECTRÓNICA } & 39 & 18 & 21 & 46 \\ \text { LENGUA MODERN } & 44 & 16 & 28 & 36 \\ \text { PSICOLOGİA } & 42 & 15 & 27 & 36 \\ \text { DERECHO } & 43 & 15 & 28 & 35 \\ \text { COMUNICACIÓN SOC } & 47 & 13 & 34 & 28 \\ \text { PEDAGOÍA INFANTIL } & 39 & 13 & 26 & 33 \\ \text { ENFERMERÍA } & 45 & 10 & 35 & 22 \\ \text { INGE. PETRÓLEOS } & 41 & 9 & 32 & 22 \\ \text { MEDICINA } & 36 & 7 & 29 & 19 \\ \text { TOTAL } & 754 & 309 & 445 & 41\end{array}$

CUADRO 01. DESERCIÓN Y RETENCIÓN USCO 2002-2005

Fuente: Registro y Control Universidad Surcolombiana

Se puede apreciar como en los primeros tres semestres se presentan los índices más altos de deserción, pues la suma de estos corresponde al $77.8 \%$, cifra compatible con la tendencia general de los estudios que se han hecho sobre deserción universitaria en América Latina, donde se corrobora que es en los tres primeros semestres en donde se produce el mayor porcentaje de abandono, cercano al $80 \%$.

En el caso de la USCO, el primer semestre es el más crítico $(41.3 \%$ ) (Ver cuadro 02), esto indica que el mayor número de los "primíparos" ingresan a la Universidad sin una claridad en la vocación profesional, ya que según este estudio, solo el $2 \%$ de los desertores recibió orientación por parte de un profesional (ver gráfica 8). Además, la precaria situación económica, pues la mayoría son de estratos 1, 2 y 3 (Ver gráfica 3) les dificulta la terminación de sus estudios al no tener ninguna proyección de cómo se van a mantener económicamente en el transcurso de la carrera. A continuación se describe el fenómeno de la deserción estudiantil por programas.

\section{FACTORES DE LA DESERCION ESTUDIANTIL EN LA USCO}

De la encuesta "Factores de la Deserción Estudiantil en la Universidad Surcolombiana 2002-2005", aplicada a 120 de los 309 desertores de los 17 programas de las 7 facultades de la USCO se obtuvo una primera 
información expuesta en la cuadro 03, gráfica 1; y que al contrastarla con los planteamientos del marco teórico de la investigación, constatan que en la deserción estudiantil de la USCO convergen tanto factores externos a la institución (socioeconómicos y personales) como factores internos a la misma (institucionales y académicos).

En efecto, los factores de índole socioeconómica (bajos ingresos familiares, desempleo, falta de apoyo familiar, incompatibilidad estudio-trabajo) inciden en la deserción en el $42.5 \%$. Los factores personales (desmotivación, problemas emocionales, desadaptación, insatisfacción académica, problemas de salud) inciden en el $39.2 \%$, pero que en conjunto constituyen los factores catalogados como externos con una incidencia total del $81.7 \%$.

Si se compara esta cifra con las que reflejan los factores académicos (bajo rendimiento, repitencia, elección inadecuada de la carrera) que es de $10.8 \%$, y los factores institucionales (cambio de institución y unificación de programas) con $7.5 \%$, que sumados representan el $18.3 \%$ sobre el total de la incidencia del fenómeno de la deserción, se tendría una tasa de incidencia cinco veces mayor de los factores procedentes del entorno institucional sobre los factores internos a la institución.

\begin{tabular}{lrr}
\multicolumn{1}{c}{ Factor de Deserción } & Frecuencia & $\%$ \\
Personales & 47 & 39,2 \\
Académicos & 13 & 10,8 \\
Socio-económicos & 51 & 42,5 \\
Institucionales & 9 & 7,5 \\
Total & 120 & 100
\end{tabular}

CUADRO 03 FACTORES DE DESERCIÓN ESTUDIANTIL USCO.

Fuente: Encuesta aplicada a desertores por el grupo investigador.

De acuerdo a la información anterior, la deserción estudiantil en la USCO se produce por factores predominantemente externos, cuyas causas están constituidas por factores socio-económicos y personales.

\section{FACTORES SOCIO- ECONÓMICOS DE DESERCIÓN}

Los factores socio-económicos (que equivalen al $42.5 \%$ de la deserción total) se discriminan de la siguiente manera: Bajos ingresos familiares, $54.9 \%$, Desempleo cabeza de familia, $25.5 \%$, Incompatibilidad entre trabajo y estudio, $14.9 \%$, Falta de apoyo familiar, $5.9 \%$.

Dentrodelosfactoressocio-económicosseconstruye una nueva categoría: Situación económica precaria del estudiante, (bajos ingresos familiares, 54.9\%, desempleo, $25.5 \%$ e incompatibilidad entre trabajo y estudio, $14.9 \%$ ), como las causas primordiales del abandono estudiantil en la USCO.

La situación económica precaria del estudiante desertor se ratifica por el estrato social del que procede, en efecto: $48.3 \%$ son de estrato $2,36.7 \%$ se les ubicó en el estrato 3; el $10.8 \%$ son de estrato 1 , y solo el $4.2 \%$ corresponden al estrato 4 .

Otro elemento a tener en cuenta es que el sostenimiento económico de los desertores depende ostensiblemente de su familia $(86.7 \%)$, y como los estratos 1 y 2 dependen mas de una economía informal o empleo disfrazado, sus ingresos además de ser exclusivamente para subsistir, no son constantes, de modo que siempre están expuestos a la incertidumbre de no generar los ingresos suficientes.

Cuando las economías familiares son frágiles es difícil pretender un apoyo económico sostenible a lo largo de toda la carrera para el mantenimiento del estudiante, pues las necesidades de la familia priorizan el trabajo al estudio. De ahí que la falta de apoyo familiar ( $5.9 \%$ ) tenga que ver directamente con la situación de precariedad antes analizada.

\section{FACTORES PERSONALES DE DESERCIÓN}

Los factores personales (que inciden en el $39.2 \%$ sobre la deserción total) se expresan de la manera siguiente: Desmotivación, 51.1\%, Insatisfacción académica, 21.3\%, Problemas de salud, $8.5 \%$, Desadaptación, $6.4 \%$, Problemas emocionales, $4.3 \%$, Otras, $8.5 \%$. 
Se puede afirmar que dentro de estos factores prima un elemento emocional, que da origen a otra nueva categoría: malestar personal $(78.8 \%)$, en la cual convergen, la desmotivación, la insatisfacción académica y la desadaptación que reflejan una indisposición y una desesperanza del estudiante que no se siente a gusto con lo que estudia, con la institución e incluso con los compañeros y docentes, lo cual ocasiona unos niveles bajos de comprensión, desinterés y apatía. Malestar que se manifiesta por el impacto que genera el paso del colegio a la universidad y que crea inseguridad y dificultades de socialización con los pares, así como la dificultad de acomodarse a modelos pedagógicos diferentes a los de la educación media secundaria.

Jewsbury y Haefeli (2000) afirman que la deserción no es un "quiebre instantáneo", supone una conflictividad del estudiante con su entorno, así el que abandona se siente abandonado primero por la institución. De modo que la decisión voluntaria de desertar está influenciada por factores académicos y psicológicos determinados, según Páramo y Correa (1999) por los actores de la deserción (docentes, compañeros, administrativos) de la universidad.

Un segundo elemento se refiere a las enfermedades del estudiante $o$ salud psicosomática (12.8\%), constituido por problemas de salud $y$ emocionales $y$ que llevan al estudiante a ensimismarse en aspectos personales y que terminan perjudicándolo en su desarrollo escolar. En este sentido los estudiantes manifestaron la necesidad de una ayuda psicológica e incluso siquiátrica que la universidad nunca asumió.

\section{FACTORES ACADEMICOS DE DESERCIÓN}

Representan el $10.8 \%$ como causales determinantes de la deserción y se discriminan como aparece a continuación: Bajo rendimiento, $46.1 \%$, Elección inadecuada de la carrera, $38.5 \%$, Repitencia, $15.4 \%$.

Estos factores se pueden clasificar en dos categorías: una nombrada falta de disciplina académica dada por el bajo rendimiento académico y la repitencia, que se explica por las insuficientes competencias lectoras $y$ escriturales de la mayoría de los desertores, el empleo inadecuado de métodos de estudio y el mal manejo del tiempo para estudiar, factores que suman el $61.5 \%$ de deserción a nivel académico en la USCO. Esto se puede entender a partir de las dificultades que tienen los estudiantes de aceptarse como actores de su propia formación, de modo que son sujetos con una percepción baja de sus capacidades y con un control externo en el logro de las metas académicas, aspecto que los lleva a tener poca credibilidad en si mismos y condicionar su fracaso académico al profesor, la institución, la suerte, y otros.

La otra categoría que aparece es la falta de orientación profesional que se determina por la elección inadecuada de la carrera, 38.5\%; pues se aprecia que el $53.3 \%$ de los desertores manifiesta no haber recibido ningún tipo de asesoría sobre la carrera a estudiar; el $25 \%$ recibió asesoría de la familia, lo cual no garantiza una calidad en la orientación. Solamente el $2.5 \%$ recibió asesoría profesional.

\section{FACTORES INSTITUCIONALES DE DESERCIÓN}

Los factores institucionales que inciden en el $7.5 \%$ sobre la deserción total, se expresan de la manera siguiente: Baja calidad académica, $55.6 \%$, Cambio de institución, $22.2 \%$, Unificación de programas, $22.2 \%$.

El factor de baja calidad académica es el de mayor incidencia en la decisión del estudiante de abandonar sus estudios y tiene relación con la inflexibilidad institucional y académica que presenta la Universidad Surcolombiana, caracterizada por currículos rígidos, prácticas docentes obsoletas, autoritarias y verticales, junto a espacios pedagógicos inadecuados (bibliotecas, laboratorios, aulas, aulas multimediales, auditorios, entre otras), que no distingue de manera alguna a sus estudiantes 
como parte fundamental de su desarrollo, pues no existe una preocupación por su historia de vida, esta no pasa de ser un registro informático de su base de datos, y como afirma Jewșbury A, y Haefeli I "las puertas están abiertas para ingresar y para salir" y la Universidad no detecta quien se queda o quien se va.

\section{CONCLUSIONES}

1- La deserción universitaria no es sólo un problema del estudiante; si bien es cierto que se asume que toda la responsabilidad recae sobre el desertor, el fenómeno de la deserción es inherente a la vida estudiantil, a situaciones económicas y socio-culturales del entorno. En este sentido el fenómeno de la deserción persistirá de alguna manera así cambien las instituciones universitarias. Conocer el problema permite establecer soluciones que controlen parcialmente los altos índices de deserción y logren mantener una tendencia creciente en la retención de estudiantes.

2- En el caso de la Universidad Surcolombiana, el mayor índice de deserción se da por factores externos a la misma, el principales factor es Socio-económico (42.5\%), el siguiente en orden de importancia se refiere al Factor personal con el $39.2 \%$. Sobre el primero es poco la incidencia que pueden ejercer las políticas universitarias tendientes a disminuir el problema; sin embargo, esto no significa que la Universidad se deba desentender del posible desertor, antes por el contrario debe implementar estrategias que permitan detectar con antelación estudiantes con alto riesgo de desertar.

Respecto al segundo factor externo que se expresa en la desmotivación e insatisfacción académica, la Universidad puede abordarlos de una manera parcial a partir de la implementación de programas que busquen estimular la pertinencia y pertenencia del estudiante con la Universidad.

Con relación a los Factores internos de deserción estudiantil, Académicos $(10.8 \%)$ e Institucionales (7.5\%), la Universidad tiene toda la responsabilidad en su solución.
Estos en conjunto inciden en un $18.3 \%$ sobre el fenómeno de la deserción en la Universidad Surcolombiana, y su incidencia es ostensiblemente menor que los factores externos expuestos anteriormente; lo cual confirma que no toda la responsabilidad de la deserción recae sobre la Institución como tal y menos aun en el desertor.

3- El problema sobre Deserción Estudiantil en la Universidad Surcolombiana sede Neiva. 2002-2005 demuestran que existe una deserción del $41 \%$ que está por debajo del índice de deserción estudiantil a nivel Nacional que es aproximadamente del $50 \%$, como señala el estudio del Ministerio Nacional de Educación en donde se afirma que entre las promociones de 1999 al 2004 , solo el $48 \%$ finalizaron sus estudios; constituye un obstáculo determinante de un alto grado de disfuncionalidad institucional que se expresa en el cumplimiento parcial de los objetivos institucionales insertos en su Misión y se explican por la inadecuación entre los fines que se propone la universidad y los medios con que cuenta para realizarlos.

4- Dentro del factor socio-económico se construye una nueva categoría: Situación Económica-Social Precaria del Estudiante, resultante de la convergencia de las variables bajos ingresos familiares, el desempleo y la incompatibilidad entre trabajo y estudio; como la causante principal del abandono estudiantil en la Universidad Surcolombiana.

El malestar personal corresponde a otra categoría, dentro de los factores personales, la cual se conforma por situaciones sicológicas disfuncionales que obstaculizan el logro de los objetivos académicos, como la desmotivación, la insatisfacción académica y la desadaptación, que reflejan una indisposición y una desesperanza del estudiante que no se siente a gusto con lo que estudia, con la institución e incluso con los compañeros y docentes, lo que lleva a éste a unos niveles bajos de comprensión, desinterés y apatía. Categoría que también tiene una incidencia significativa en la deserción estudiantil.

5- La Universidad Surcolombiana a pesar de sus 
limitaciones institucionales y su relativamente alto índice de Deserción Estudiantil, por su prestigio, por su nivel académico, por ser pública y la menos costosa en la región, sigue siendo la primera opción y casi la única para egresados de la educación media básica de colegios oficiales de los estratos 1 , 2 y 3.

\section{RECOMENDACIONES}

1. Es necesario revisar y ajustar los procesos de selección y admisión de los aspirantes a ingresar a la universidad para detectar tempranamente los potenciales desertores y hacer el respectivo seguimiento de su trayectoria académica con el fin de aplicar medidas pertinentes hacia aquellos que presentan mayores dificultades, mediante la instauración de políticas de apoyo socio-económico y emocional; complementariamente adoptar practicas pedagógicas que respondan mas eficazmente al tipo de estudiante USCO.

2. Un objetivo prioritario de Bienestar Universitario de la Universidad Sur Colombiana será reducir los índices de deserción estudiantil, minimizando los efectos de la precariedad socio-económica y el malestar personal del estudiante. Con respecto a la precariedad se buscará vincular con remuneración a los mejores estudiantes, de los sectores más vulnerables a la deserción, como asistentes de docencia, monitores de laboratorios y deportivos, ayudantes de biblioteca, auxiliares de investigación, integrantes de grupos artísticos, entre otros. De igual manera la Universidad debe ampliar y mejorar los servicios de asistencia estudiantil (servicio de restaurante, servicios médicos y odontológicos, recreación y deportes).

En cuanto al malestar personal una atención psicológica, una consejería confiable y una comunicación mas eficiente y humana con docentes, directivos y administrativos de la Universidad Surcolombiana, coadyuvará al estudiante a una mejor adaptación al ambiente universitario.

3-Mejorar la eficiencia en la gestión administrativa, pues la cultura universitaria en la USCO da prioridad a la administración sobre la academia. Esto conduce a una carencia de espacios académicos y culturales adecuados, una deficiente infraestructura física, una permanente falta de disponibilidad en materiales y equipos educativos que dificultan la interacción entre los miembros de la comunidad universitaria lo cual condiciona al estudiante a carecer de identidad con la universidad y lo predispone a la desercion.

\section{BIBLIOGRAFÍA BÁSICA}

ÁLVAREZ, José María (1997) Etiología de un sueño. Universidad Autónoma de Colombia. Bogotá.

FUNDACIÓN RESTREPO BARCO (2003) Situación de la educación básica, media y superior en Colombia. Bogotá.

JEWSBURY, A; HAEFELI, I (2002). Análisis de la deserción en universidades públicas argentinas. PÁRAMO, Gabriel; CORREA, Carlos (1999). Deserción Estudiantil Universitaria. Conceptualización. En: Revista Universidad EAFIT, Abril - Mayo - Junio, Medellín, 1999.

El problema de la desercion escolar, en: Revista de la Contraloría General de la Republica. Noviembre-Diciembre 2005. Edición 311.

RINCÓN, Cecilia y otros (2004) Deserción y retención escolar. Magisterio-Universidad Francisco José de Caldas. Bogotá.

UNIVERSIDAD NACIONAL DE COLOMBIAICFES. (2002) Estudio de la deserción estudiantil en la educación superior en Colombia. Bogotá.

UNIVERSIDAD SURCOLOMBIANA (2002). Boletín Estadístico 2001-2002. Neiva.

UNIVERSIDAD SURCOLOMBIANA (2005) Boletín Estadístico 2003-2004. Neiva.

www.mineducacion.gov.co. La deserción estudiantil: reto investigativo y estratégico asumido de forma integral por la UPN. 